\title{
Research on fixed pattern noise de-noising of APS COMS
}

\author{
Zheng Tuo, Quan Haiyang
}

Beijing Microelectronics Technology Institute, Beijing 100076, China;

2217404576@qq.com

Keywords: de-noising; star sensor;

\begin{abstract}
Star sensor is an important part of the attitude control of a space vehicle. At the time of image acquisition, CMOS sensors will have some fixed pattern noise which will affect the image capture effects. From the practical application, this article apply the average of de-noising algorithm to eliminate the effects of noise caused by fixed pattern, which can effectively improve the accuracy of image capture, thus helping centroid localization be more accurate.
\end{abstract}

\section{Introduction}

As a product of the development of optical remote sensing technology, star sensor provides an important help for spacecraft's attitude determination. In the early 90 century, CCD technology has been widely used. The CCD has many advantages, such as inherent high sensitivity, wide spectral response, low readout noise and large dynamic range and other advantages, which makes it become the most common imaging device. But with the expanding application range, its disadvantages are gradually revealed. The volume, weight and power consumption of CCD is large. What's worse, since it is very sensitive to the cosmic radiations, it will seriously affect the accuracy. Meanwhile, CMOS sensor technology uses active pixel sensor technology, making it become the focus of the design of star sensor, and get rapid progress. Although CMOS APS has some advantages such as high integration degree, anti radiation, power supply and simple drive circuit, its sensitivity is lower while its noise is higher when compared with CCD. In fact, the noise will affect the accuracy of subpixel centroid localization algorithm, so it is necessary for us to study it. [1-2]

\section{Centroid method}

The centroid method uses the centroid of the spot signal as the feature point of spot position and use the centroid algorithm to find the centroid signal location. The calculation is easy to conduct. So it is widely used to estimate the location of the target image points. When the target image points are distributed in a matrix window size is $m \times n$ and the size is represented as $f(x, y)$. We need to segment the original image through threshold. $\mathrm{V}$ indicates the background threshold while centroid is actually the first moment of the image after calculating the binarization.

The centroid method can be regarded as a kind of weighted calculation of the gray value of the target image. The closer the image is to the center of the star, the greater the weight is. In this case, it also can represent the specific location of the gray center better. On this basis, we can derive more accurate algorithm, such as: the centroid method with the threshold, square weighted centroid method and so on. But regardless of any kind of method, calculating the centroid of the diffused star will depend on too much weak signal of both sides of the peak value, which just results in the weakness of anti noise interference ability of the centroid method. In order to enhance its anti-jamming ability, the corresponding de-noising algorithm is needed to be proposed.[3]

\section{Fixed pattern noise in APS devices}

There are many kinds of noise in APS devices. The FPN (Fix Pattern Noise) will have an important impact on the imaging quality. It is mainly caused by the MOS FET threshold voltage deviation, pixel endogenous follower device gain errors, transistor size deviation, amplifier gain and bias and deviation of the reset line impedance leads to the reset voltage deviation caused by the 
column. The equivalent number of equivalent electrons with fixed pattern noise is, and the root mean square value of the noise is:

It has been able to remove or suppress fixed pattern noise to through some new techniques; the most commonly used method is the correlated double sampling circuit. Although appropriate measures are taken, in the middle and high level applications, there will still be some important impact.

In calculating the centroid, because of noise a pixel (I, J) output signal should include two parts: the real star signal and noise signal .Then , . Due to the presence of the actual noise, the centroid position error can be expressed as:.So when the size of the dispersed spot centroid calculation selection increases, the noise will impact on the centroid computation [4]

\section{Mean value de-noising}

In this paper, a domestic APS CMOS sensor, whose pixel size of $15 \mathrm{um}$, resolution $1024 \times$ 1024 will be introduced.

In the condition when the image is full black, because of the existing fixed noise, the picture cannot show all black state. In the state when the pixel maximum reaches 255 (0xff), the noise will be collected in pictures with no regularities. And the average value is about 9 (0x09), which will have an effect on collecting star spots and calculating the centroid. When using the sub pixel centroid calculation, the influence of the fixed pattern noise will lead to the error in the final calculation. Due to the irregular presentation of fixed pattern noise, its average value can be taken as a fixed system noise. Then through seeking the difference between the acquisition of the image and the average value, preliminary de-noising effect can be obtained.[5]

Due to different integration time, the fixed pattern noise will be slightly different. According to calculate the data when the the integral time is $10 \mathrm{~ms}, 50 \mathrm{~ms}, 100 \mathrm{~ms}$ respectively and use the method of 10 times the average value, the difference of average noise and the actual noise can be obtained, with abscissa as the gray value, longitudinal coordinates for the frequency of occurrence. The results are as follows. 

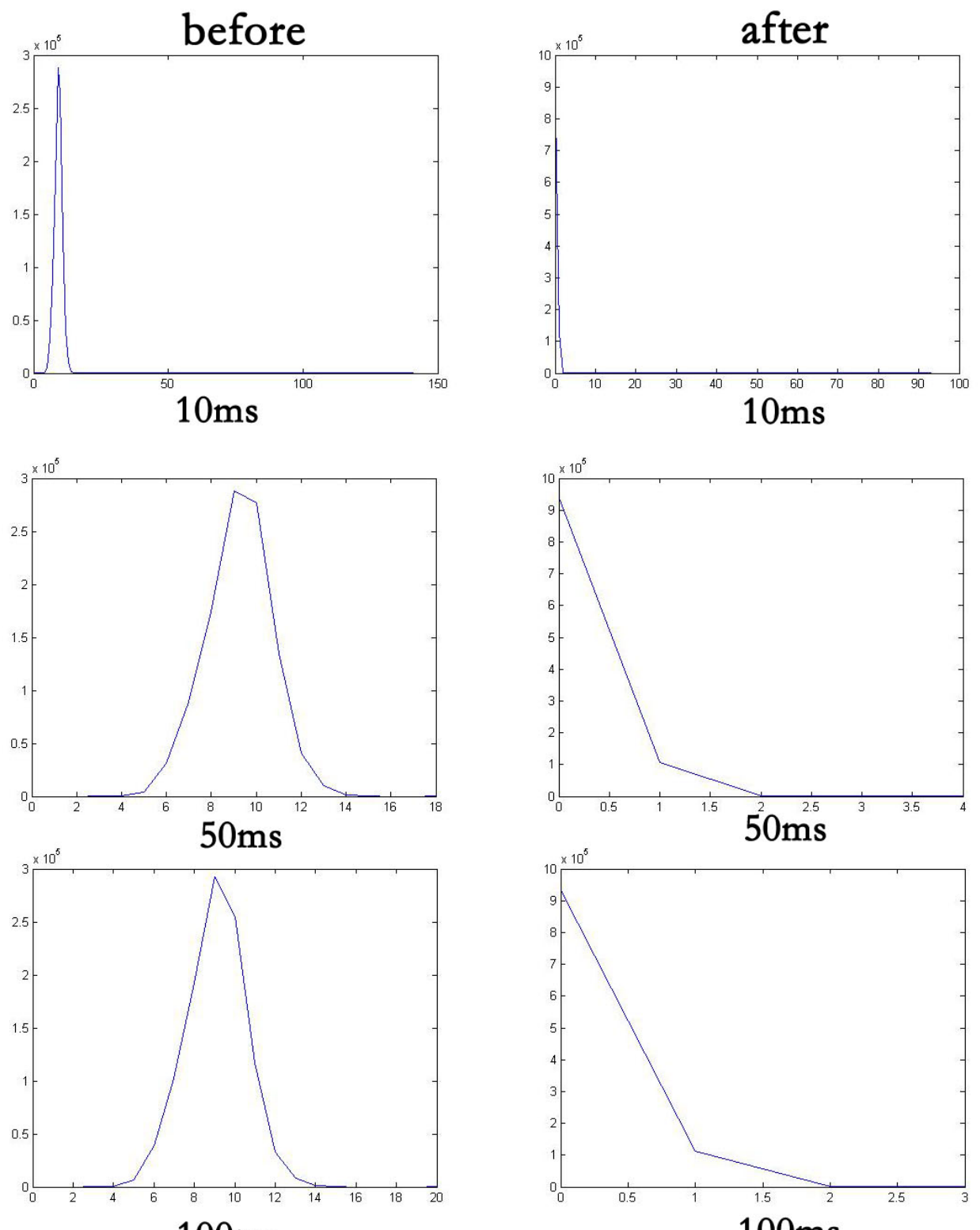

$100 \mathrm{~ms}$

$100 \mathrm{~ms}$

Fig. 1 the gray value change before and after de-noising

\section{Results Analysis}

In the collection of the full black picture, the fixed noise interference will bring about $3.6 \%$ of the impact. The gray value of noise concentration is about $08-10$. When the average value is used to de-noising method, the gray value of the noise is concentrated in the 00-02.

Table 1 the gray value change before and after de-noising

\begin{tabular}{|c|c|c|}
\hline integration time & $\begin{array}{c}\text { Average noise before } \\
\text { de-noising }\end{array}$ & $\begin{array}{c}\text { Average noise after } \\
\text { de-noising }\end{array}$ \\
\hline $10 \mathrm{~ms}$ & 9.2131 & 0.1424 \\
\hline $50 \mathrm{~ms}$ & 9.2414 & 0.1041 \\
\hline $100 \mathrm{~ms}$ & 9.0757 & 0.1102 \\
\hline
\end{tabular}



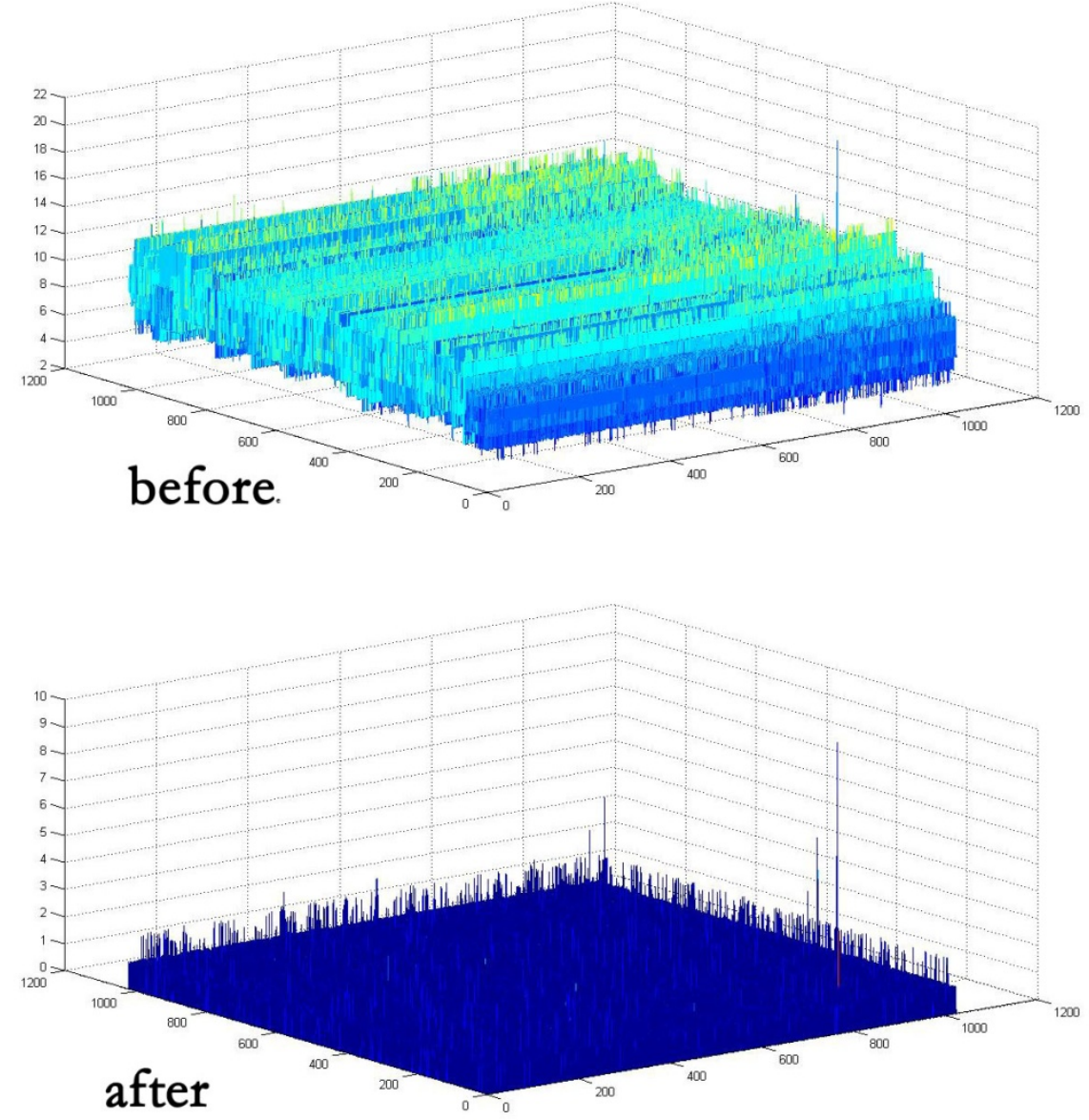

Fig.2 the gray value change before and after de-noising

It can be seen that noise interference reduced to less than $0.05 \%$ after de-noising. It can be considered that the interference of fixed noise is eliminated.

\section{Conclusions}

This paper theoretically analyzes the fixed noise influence on image and according to the specific parameters of a domestic CMOS APS sensors, using average de-noising method to remove noise, significantly reduced the fixed noise effects. The centroid location can provide higher precision of image processing effect and help the centroid location more accurate.

\section{Reference}

[1-2]Li Jie. Research on the key technology of APS star sensor. [D]. Changchun: 2005:8-9.

[3] Wu Feng. Research on the key technologies of autonomous navigation star sensor [D]. Suzhou: Soochow University, 2012:3-4.

[4] Shi Shaolong, Yin Dayi. CMOS APS Noise effect on position accuracy of star tracker. [J].Beijing 2013

[5] Zhi Shuai, Zhang Lin, Li Xinlu. Realization of simulated star map with noise. [J].Changchun:2014 